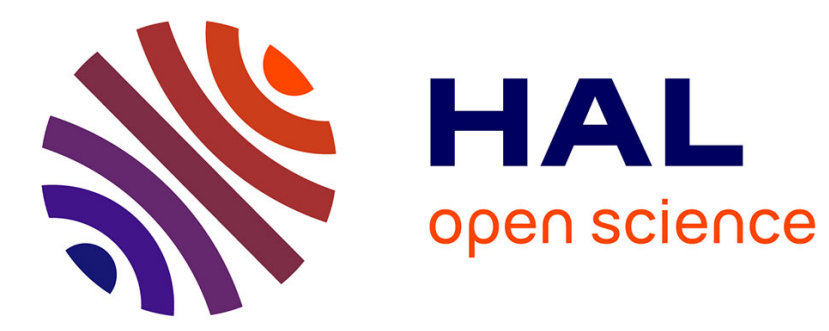

\title{
Metric Tensor for Multicomponent Edge Detection
} Sylvain Rousseau, David Helbert, Philippe Carré

\section{To cite this version:}

Sylvain Rousseau, David Helbert, Philippe Carré. Metric Tensor for Multicomponent Edge Detection. ICIP, Sep 2010, Hong-Kong, Hong Kong SAR China. pp.2. hal-00493428

\section{HAL Id: hal-00493428 \\ https://hal.science/hal-00493428}

Submitted on 20 Dec 2013

HAL is a multi-disciplinary open access archive for the deposit and dissemination of scientific research documents, whether they are published or not. The documents may come from teaching and research institutions in France or abroad, or from public or private research centers.
L'archive ouverte pluridisciplinaire HAL, est destinée au dépôt et à la diffusion de documents scientifiques de niveau recherche, publiés ou non, émanant des établissements d'enseignement et de recherche français ou étrangers, des laboratoires publics ou privés.

$$
\text { Copyright }
$$




\title{
METRIC TENSOR FOR MULTICOMPONENT EDGE DETECTION
}

\author{
Sylvain Rousseau*, David Helbert, Philippe Carré \\ XLIM, CNRS, University of Poitiers, BP 3017986962 Futuroscope Chasseneuil - France \\ rousseau@sic.univ-poitiers.fr
}

\begin{abstract}
In this paper, we present the use of differential geometry for the segmentation of multispectral images, which allows us to unify several known methods including projecting onto a particular axis or a particular plan. This is done by choosing a metric tensor on the feature space computing the pullback of the metric tensor and applying standard Di Zenzo algorithm.
\end{abstract}

Index Terms - metric tensor, Riemannian manifold, metric pullback

\section{INTRODUCTION}

Edge detection is a basic step for image understanding and computer vision. Since abrupt gray-level changes occur at edge points, most edge detection algorithms use image gradient information. Edge detection for color image presents some challenges. The common technique is to look for discontinuities in luminance component and while ignoring chrominance information.

This paper proposes to review and to extend multicomponent classical method with differential geometry. This method contains two steps: metric tensor caculation and edge detection with Di Zenzo's algorithm. The choice of a particular metric tensor on the space feature will induce different edge detection methods. The synthetic image we use serve as a proof-of-concept.

The outline of the paper is as follows. Section 2 reviews the classical approach of the gradient. Section 3 describes the Riemannian tools used to detect particular edges, and various color difference equations. Section 4 shows the performance of the edge detection algorithm. Section 5 presents concluding remarks.

\section{THE CLASSICAL POINT OF VIEW}

A gradient of a multi-image introduced in [1] relies on some tools of differential analysis. A $m$-dimensional image is indeed proposed to be viewed as a map $f$ from a domain $D \subset$ $\mathbb{R}^{2}$ to the so called space feature $\mathbb{R}^{m}$. The set $\varphi(D)$ is then

\footnotetext{
*We would like to thank Jacques Blanc-Talon Head of Scientific Domain "Information Technology" of DGA.
}

a sub-manifold of $\mathbb{R}^{m}$ provided that the Jacobian is of rank 2 everywhere and that $f$ is injective.

$$
\begin{aligned}
& f: \quad D \quad \longrightarrow \quad \mathbb{R}^{m} \\
& (x, y) \longmapsto\left(f^{1}(x, y), \ldots, f^{m}(x, y)\right)
\end{aligned}
$$

Let $f_{1}=\left(\frac{\partial f^{1}}{\partial x}, \ldots, \frac{\partial f^{m}}{\partial x}\right)$ and $f_{2}=\left(\frac{\partial f^{1}}{\partial y}, \ldots, \frac{\partial f^{m}}{\partial y}\right)$. A tensor $T$ on $\varphi(D)$ is then introduced:

$$
T=\left(\begin{array}{ll}
\left\|f_{1}\right\|^{2} & f_{1} \cdot f_{2} \\
f_{1} \cdot f_{2} & \left\|f_{2}\right\|^{2}
\end{array}\right)
$$

which can be written

$$
J^{T} J \quad \text { where } \quad J^{T}=\left(\begin{array}{ccc}
\frac{\partial f^{1}}{\partial x} & \cdots & \frac{\partial f^{m}}{\partial x} \\
\frac{\partial f^{1}}{\partial y} & \cdots & \frac{\partial f^{m}}{\partial y}
\end{array}\right) .
$$

Edges of the image are detected by maximizing this tensor. We remark that these tensors given on each point of the image define no more than a metric on the domain $D$.

The function $f$ can be slightly modified as pointed out in [2] by adding two space coordinates:

$$
\begin{array}{cccc}
\varphi: & D & \longrightarrow & \mathbb{R}^{m+2} \\
(x, y) & \longmapsto & \left(x, y, \varphi^{1}(x, y), \cdots, \varphi^{m}(x, y)\right)
\end{array}
$$

The application $\varphi$ is then an embedding.

\section{RIEMANNIAN TOOLS}

\subsection{Concepts}

A mathematical approach can be seen in $[3,4]$ where Riemannian tools are used. We still have the embedding $\varphi$ but the metric tensor $T$ is viewed as the pullback of a metric tensor $g$ defined on $\mathbb{R}^{m+2}$. Let us recall some mathematical facts. Let $M^{m}$ and $N^{n}$ two Riemannian manifold and $\varphi: M^{m} \longrightarrow N^{n}$ an embedding of $M^{m}$ in $N^{n}$. Given a (pseudo-)Riemannian metric $g$ on $N$, we can construct one on $M$ called the pullback of $g$ and denoted $\varphi^{*} g$. The expression of $\varphi^{*} g$ is given by:

$$
\varphi^{*} g=J^{T} g J
$$

where $J$ is the Jacobian of $\varphi$. 


\subsection{Application with various metrics}

In this section, we will show that choosing a particular uniform metric on the space feature will induce different known methods.

Suppose $m=1$, we have a grey-level image of intensity I. Then,

$$
\varphi: \begin{array}{ccc}
D & \longrightarrow & \mathbb{R}^{3} \\
(x, y) & \longmapsto & (x, y, I(x, y))
\end{array}
$$

and

$$
J=\left(\begin{array}{cc}
1 & 0 \\
0 & 1 \\
I_{x} & I_{y}
\end{array}\right)
$$

where $\frac{\partial I}{\partial x}$ is denoted by $I_{x}$ and $\frac{\partial I}{\partial y}$ by $I_{y}$. If $\mathbb{R}^{3}$ is endowed with the (pseudo-)metric,

$$
g=\left(\begin{array}{lll}
0 & 0 & 0 \\
0 & 0 & 0 \\
0 & 0 & 1
\end{array}\right)
$$

then we get:

$$
\varphi^{*} g=\left(\begin{array}{cc}
I_{x}^{2} & I_{x} I_{y} \\
I_{x} I_{y} & I_{y}^{2}
\end{array}\right)
$$

which is the metric tensor on $D$ obtain by Di Zenzo. This tensor is then obtained by choosing the metric tensor

$$
\left(\begin{array}{lll}
0 & 0 & 0 \\
0 & 0 & 0 \\
0 & 0 & 1
\end{array}\right)
$$

on the space feature.

Suppose now the case of a color image with $m=3$ :

$$
\begin{aligned}
& \varphi: D \quad \longrightarrow \quad \mathbb{R}^{5} \\
& (x, y) \longmapsto\left(x, y, I^{1}(x, y), I^{2}(x, y), I^{3}(x, y)\right)
\end{aligned}
$$

where $I^{1}, I^{2}, I^{3}$ correspond respectively to the red, green and blue component of the image. Different algorithms will be applied to the synthetic image 1 :

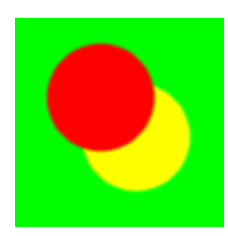

Fig. 1. Synthetic test image.
We can perform marginal methods simply by choosing a metric of the form:

$$
g=\left(\begin{array}{lllll}
0 & 0 & 0 & 0 & 0 \\
0 & 0 & 0 & 0 & 0 \\
0 & 0 & 1 & 0 & 0 \\
0 & 0 & 0 & 0 & 0 \\
0 & 0 & 0 & 0 & 0
\end{array}\right)
$$

so

$$
\varphi^{*} g=\left(\begin{array}{cc}
\left(I_{x}^{1}\right)^{2} & I_{x}^{1} I_{y}^{1} \\
I_{x}^{1} I_{y}^{1} & \left(I_{y}^{1}\right)^{2}
\end{array}\right)
$$

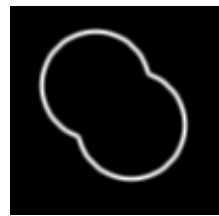

Fig. 2. Di Zenzo algorithm with metric (1).

In this case, we are only looking at the red component so there no distinction between red and yellow.

In this example, we are interested in another particular direction that is the grey axis. This is done by choosing:

$$
g=\left(\begin{array}{lllll}
1 & 0 & 0 & 0 & 0 \\
0 & 1 & 0 & 0 & 0 \\
0 & 0 & 1 & 1 & 1 \\
0 & 0 & 1 & 1 & 1 \\
0 & 0 & 1 & 1 & 1
\end{array}\right)
$$

The pullback metric is:

$$
\varphi^{*} g=\left(\begin{array}{cc}
1+\sum_{i=1}^{3}\left(I_{x}^{i}\right)^{2} & \sum_{i=1}^{3} I_{x}^{i} I_{y}^{i} \\
\sum_{i=1}^{3} I_{x}^{i} I_{y}^{i} & 1+\sum_{i=1}^{3}\left(I_{y}^{i}\right)^{2}
\end{array}\right),
$$

which give the following expected result: edges between region with same luminance (red and green) are not detected.

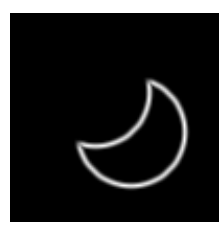

Fig. 3. Di Zenzo algorithm with metric (2).

More generally, we can choose a particular direction on which to project. Let $u=(a, b, c)^{T}$ a unit vector. Take the 
quadratic form $Q=u u^{T}$. We have $Q(u)=u^{T} Q u=1$ and $Q(v)=0$ for any vector orthogonal to $u$. The metric is then of the form:

$$
g=\left(\begin{array}{ll}
1 & 0 \\
0 & 1
\end{array}\right) \oplus\left(\begin{array}{lll}
a^{2} & a b & a c \\
a b & b^{2} & b c \\
a c & b c & c^{2}
\end{array}\right)
$$

The metric (2) can then be found up to a scalar by choosing $u=\frac{1}{\sqrt{3}}(1,1,1)^{T}$.

In the last example we choose a metric that will only take into account the chromaticity of the color. More generally we are looking for a quadratic form that is degenerate along one direction and Euclidean on the space orthogonal to that direction. Let $u$ that degenerate direction and $v, w$ so that $(u, v, w)$ is an orthonormal frame. Let $Q=v v^{T}+w w^{T}$, then $Q(u)=0$ and $Q(v)=Q(w)=1$. If we suppose $u=$ $\frac{1}{\sqrt{3}}(1,1,1)^{T}$ then:

$$
g=\left(\begin{array}{ll}
1 & 0 \\
0 & 1
\end{array}\right) \oplus \frac{1}{3}\left(\begin{array}{ccc}
2 & -1 & -1 \\
-1 & 2 & -1 \\
-1 & -1 & 2
\end{array}\right)
$$

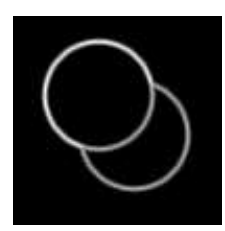

Fig. 4. Di Zenzo algorithm with metric (4).

Marginal methods, intensity methods and chromaticity methods can be performed simply by choosing a particular matrix, computing the pullback and applying the usual algorithm. The interesting thing here is that we can mix these methods and apply at a point of a given color one method and one other method at some other location.

\section{EDGE DETECTION WITH SPATIAL ADAPTATIVE METRICS}

In the previous section, we have seen some examples using uniform metrics. But metrics can be computed locally to improve edge detection. For example, we are considering in this section a metric of the form (3). The direction $u(x, y)$ is set to the first principal component of pixels located in a window of fixed size centered in $(x, y)$.

Let us consider the following synthetic image where the noise is orthogonal to the principal direction of the image.

We apply the usual algorithm corresponding to the Euclidean metric. The edge is not detected because the noise is considered by the Euclidean metric as edges.

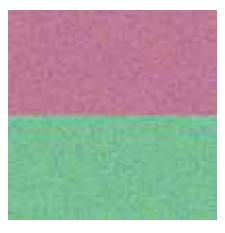

Fig. 5. Noisy image.

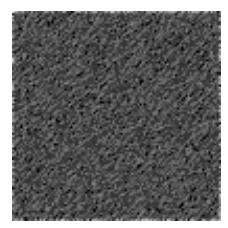

Fig. 6. Di Zenzo algorithm with Euclidean metric.

On the other hand, when we choose metric of the form (3) where $u$ is the principal direction of the image, the noise is reduced because it is orthogonal to the principal direction.

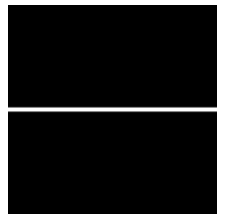

Fig. 7. Di Zenzo algorithm with SVD metric.

\section{CONCLUSION}

This paper presents a framework based on some mathematical concepts that allows us to consider simultaneously several edge detection methods. Marginal method and luminance method can be reduced to the choice of a $3 \times 3$ matrix. Different methods can be spatially combined. We have processed a synthetic image, to illustrate the potential of this framework. We would like to investigate further with hyperspectral images using a tensor metric field depending on spectral signatures.

\section{REFERENCES}

[1] S. Di Zenzo, "A note on the gradient of a multi-image," Computer Vision, Graphics, and Image Processing, vol. 33, no. 1, pp. 116-125, 1986.

[2] A. Cumani, "Edge detection in multispectral images," CVGIP: Graphical Models and Image Processing, vol. 53, no. 1, pp. 40-51, 1991. 
[3] N. Sochen, R. Kimmel, and R. Malladi, "A general framework for low level vision," IEEE Transactions on Image Processing, vol. 7, no. 3, pp. 310-318, 1998.

[4] T. Batard, C. Saint-Jean, and M. Berthier, "A metric approach to $\mathrm{nD}$ images edge detection with Clifford algebras," Journal of Mathematical Imaging and Vision, vol. 33, no. 3, pp. 296-312, 2009. 